\title{
Density of GeV Muons Measured with IceTop
}

\section{The IceCube Collaboration}

(a complete list of authors can be found at the end of the proceedings)

E-mail: soldin@udel.edu

We present a measurement of the density of $\mathrm{GeV}$ muons in near-vertical air showers using three years of data recorded by the IceTop array at the South Pole. We derive the muon densities as functions of energy at reference distances of $600 \mathrm{~m}$ and $800 \mathrm{~m}$ for primary energies between $2.5 \mathrm{PeV}$ and $40 \mathrm{PeV}$ and between $9 \mathrm{PeV}$ and $120 \mathrm{PeV}$, respectively, at an atmospheric depth of about $690 \mathrm{~g} / \mathrm{cm}^{2}$. The measurements are consistent with the predicted muon densities obtained from Sibyll 2.1 assuming any physically reasonable cosmic ray flux model. However, comparison to the post-LHC models QGSJet-II.04 and EPOS-LHC shows that the post-LHC models yield a higher muon density than predicted by Sibyll 2.1 and are in tension with the experimental data for air shower energies between $2.5 \mathrm{PeV}$ and $120 \mathrm{PeV}$.

Corresponding author: Dennis Soldin ${ }^{1 *}$

${ }^{1}$ Bartol Research Institute, Dept. of Physics and Astronomy

University of Delaware, Newark, DE 19716, USA

${ }^{*}$ Presenter

$37^{\text {th }}$ International Cosmic Ray Conference (ICRC 2021)

July 12th - 23rd, 2021

Online - Berlin, Germany 


\section{Introduction}

Cosmic rays interact in the Earth's atmosphere and produce extensive air showers (EAS) which can be measured with large detector arrays at the ground. The properties of the initial cosmic ray, such as energy and mass, are inferred indirectly from the particles measured at the ground and their interpretation strongly relies on simulations of the EAS development and thus on theoretical models [1]. One of the main challenges in understanding EAS is the description of hadronic interactions over several decades in center-of-mass energy. The relevant interactions are in the forward fragmentation region which can not be studied with existing colliders and their cross-sections cannot be computed from perturbative quantum chromodynamics. Instead, they are calculated using phenomenological models tuned to a variety of data sets from collider and fixedtarget experiments, and are extrapolated into the phase space relevant for EAS. Several hadronic interaction models are available where the most recent ones take high-energy data from the LHC into account and are thus commonly referred to as post-LHC models, such as EPOS-LHC [2] and QGSJet-II.04 [3], in contrast to older pre-LHC models, like Sibyll 2.1 [4]. Air shower experiments can test and help to improve hadronic models with measurements of the muon content in EAS.

This article reports a measurement of the density of muons with energies of around $1 \mathrm{GeV}$ at large lateral distances in EAS with energies between $2.5 \mathrm{PeV}$ and $120 \mathrm{PeV}$ with IceTop. The results will be compared to predictions from simulations based on recent hadronic interaction models and discussed in the context of different cosmic ray mass composition assumptions.

\section{IceTop}

IceTop [5] is the surface air shower detector of the IceCube Neutrino Observatory [6] which is located at an altitude of about $2.8 \mathrm{~km}$ above sea level (average atmospheric depth of $692 \mathrm{~g} / \mathrm{cm}^{2}$ ) at the geographic South Pole. It consists of 81 stations deployed in a triangular grid with a typical separation of $125 \mathrm{~m}$. Each station consists of two tanks separated by about $10 \mathrm{~m}$ which are filled with clear ice and contain two Digital Optical Modules (DOMs) which measure the Cherenkov light produced by EAS particles traversing the tanks. These stations have two readout modes: a Hard Local Coincidence (HLC) hit occurs when both tanks in a station have a discriminator trigger within a time window of $125 \mu \mathrm{s}$. If there is a discriminator trigger in only one tank, it is called a Soft Local Coincidence (SLC) hit. While for HLC hits the full wave form of the DOM signals is recorded, for an SLC hit only the integrated signal charge and a timestamp are available. The tank signals are calibrated to be expressed in units of Vertical Equivalent Muon (VEM), which is the average charge produced by a vertically through-going muon. To determine the number of muons in an EAS, only the SLC information is used because at large distances from the shower axis, where muons are expected to dominate the EAS content, mostly only one tank of a station is hit. The distribution of SLC hits for near-vertical events with reconstructed energies between $10 \mathrm{PeV}$ and 12.5 PeV as a function of lateral distance from the shower core and charge is shown in Fig. 1. While the electromagnetic shower component produces a signal distribution which approximately follows a power law over the entire lateral distance range, a characteristic structure at signals around 1 VEM produced by muons becomes visible at large distances, the so-called Muon Thumb. This population consists mostly of tanks hit by one muon, and it is used to determine the muon content in EAS. 
The HLC hits are used to determine the shower direction, the intersection point of the shower axis with IceTop (the shower core), and the shower size. This is done by fitting the measured signals with a Lateral Distribution Function (LDF) and their times with a phenomenological model of the shower front, as described in Ref. [5]. The LDF includes an attenuation factor which accounts for the snow coverage on top of each tank. The energy of the primary cosmic ray, $E_{\text {reco }}$, is estimated based on the shower size, $S_{125}$, defined as the signal at a lateral distance of $125 \mathrm{~m}$, and the true primary energy, as obtained from simulations based on Sibyll 2.1. The resulting energy resolution is better than 0.1 in $\log _{10}\left(E_{\text {reco }}\right)$ for all energies considered. Only events with reconstructed energy $E_{\text {reco }} \geq 2.5 \mathrm{PeV}$ are considered, an energy above which IceTop reaches a detection efficiency close to $100 \%$ for all cosmic ray masses, from hydrogen up to iron [7].

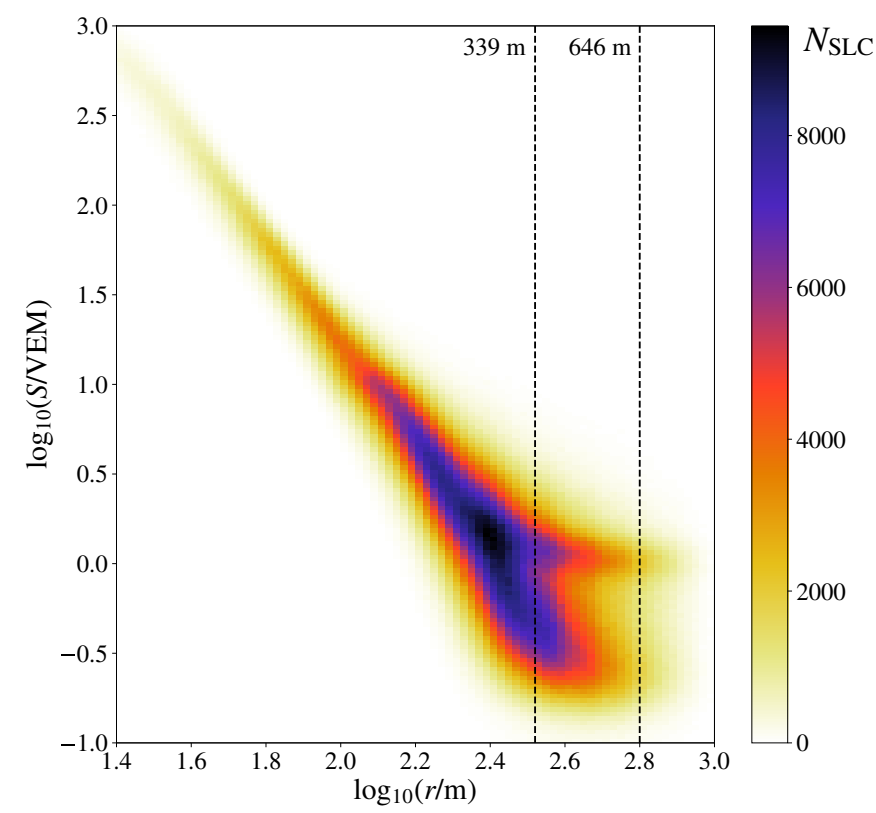

Figure 1: Distribution of SLC signals for near-vertical events $\left(\theta<18^{\circ}\right)$ with reconstructed energies between $10 \mathrm{PeV}$ and $12.5 \mathrm{PeV}$ as a function of lateral distance and charge. A characteristic structure produced by muons in IceTop tanks at large distances, the so-called Muon Thumb, is visible at signals around $1 \mathrm{VEM}$.

\section{GeV Muon Analysis}

This analysis uses data collected by IceTop between May 31, 2010 and May 2, 2013 with more than 18 million events which pass the selection criteria, corresponding to around 947 days of data acquisition. The event selection only considers events with triggers recorded in more than 5 stations and a successful EAS reconstruction. In addition, the shower core must be within the geometrical area of IceTop, the tank with the largest signal must not be at the edge of the array, and there must be at least one station with signal greater than 6 VEM. This analysis is further restricted to near-vertical events with zenith angles $\theta<18^{\circ}$ in order to select near-vertical muons.

The muon content in EAS is determined based on the characteristic Muon Thumb in the signal distribution, shown in Fig. 1. This is done using a log-likelihood method to fit the signal distributions at fixed energy, zenith, and lateral distance (i.e. slices in $r$ of Fig. 1), using a multi-component model, which is illustrated in Fig. 2 for lateral distances of about $340 \mathrm{~m}$ and $650 \mathrm{~m}$. The figures show the muon signal distribution, an empirically determined distribution of signals with no muons, and the distribution of accidental signals. Thus the model includes individual signal models for the detector response to muons, the electromagnetic (EM) part of the EAS, and the contribution from accidental coincident background hits, which are described in detail in Refs. [8-10]. As shown in Fig. 2, the muon peak becomes dominant at large lateral distances where a large fraction of the recorded SLC hits are caused by single muons traversing the IceTop tanks. 


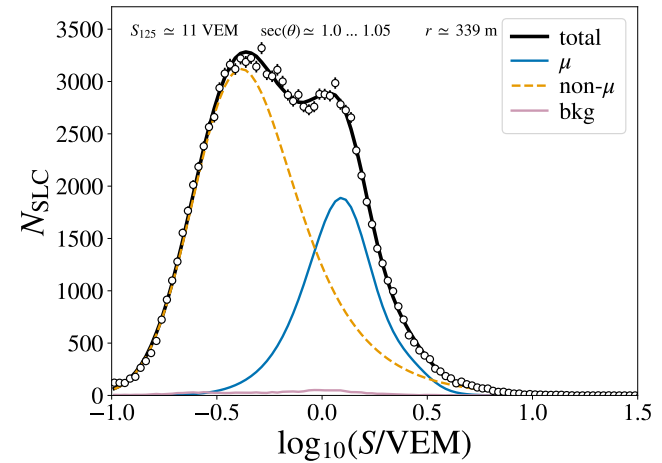

(a)

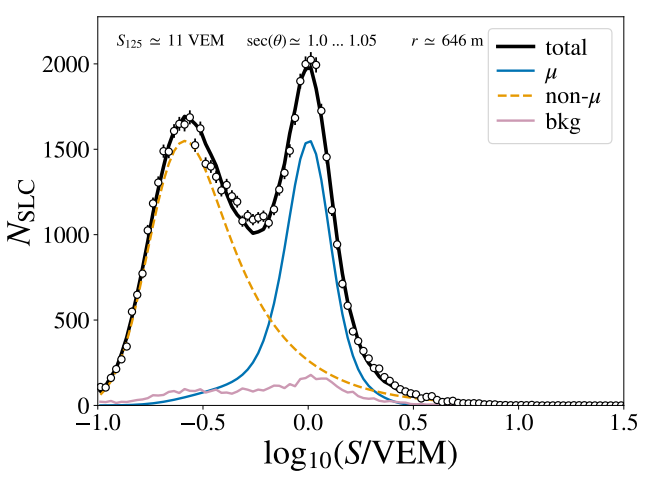

(b)

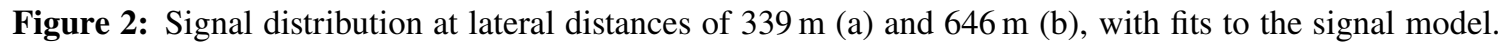
These figures correspond to vertical slices in Fig. 1. The lines show the muon signal model (blue solid), the distribution of signals with no muons (dashed yellow) and the distribution of accidental signals (pink solid).

The muon response model accounts for the trajectory of the muons through the IceTop tanks and the finite detector resolution. Although the chance for two simultaneous muon hits is very small at large radii, charge distributions for up to three simultaneous muon hits are considered. The default EM model (EMI) assumes that the signals approximately follow a power-law. However, in order to allow small deviations from a simple power-law as a function of lateral distance, a second EM model (EM2) is assumed and the differences between the models are included as systematic uncertainty in the final results. The contribution from accidental coincident background is modelled according to a Poisson distribution based on data taken in an off-time window before the EAS front arrives. The likelihood fit finds the mean number of muons per event $\left\langle N_{\mu}\right\rangle$ which is then divided by the cross-sectional area of the tanks projected onto a plane perpendicular to the shower axis to yield the muon density at a given location, $\rho_{\mu}(r)$.

The resulting raw reconstructed muon densities, $\hat{\rho}_{\mu}(r)$, are shown in Fig. 3. These distributions are fit to interpolate the raw muon densities at radial distances of $600 \mathrm{~m}$ and $800 \mathrm{~m}$. However, a small deviation of the raw reconstructed muon density from the truth is observed in simulations. This deviation is corrected by multiplying a correction factor to the raw density, $\hat{\rho}_{\mu}(r)$. The correction is determined by dividing the reconstructed muon density in simulations by the true muon density. The resulting ratios obtained from proton and iron shower simulations using CORSIKA [11] based on the hadronic interaction models Sibyll 2.1 [4], EPOS-LHC [2], and QGSJet-II.04 [3], are shown in Fig. 4. Also shown are the average ratios for these three hadronic interaction models.

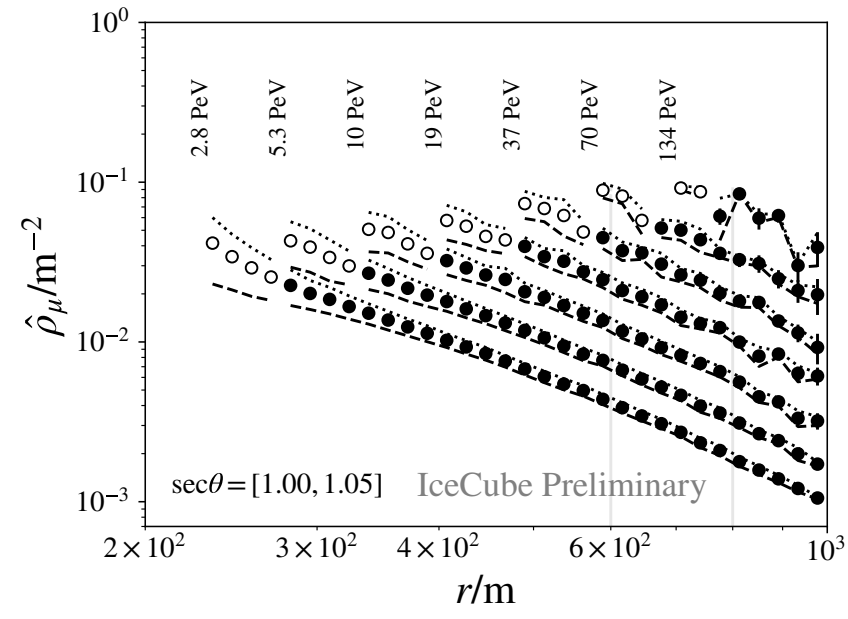

Figure 3: The raw reconstructed muon densities, $\hat{\rho}_{\mu}$, as a function of lateral distance for seven energy bins. The lines indicate the systematic uncertainty associated to the function used to model signals with no muons. Filled markers correspond to lateral distances where more than $80 \%$ of signals are SLC hits only. 

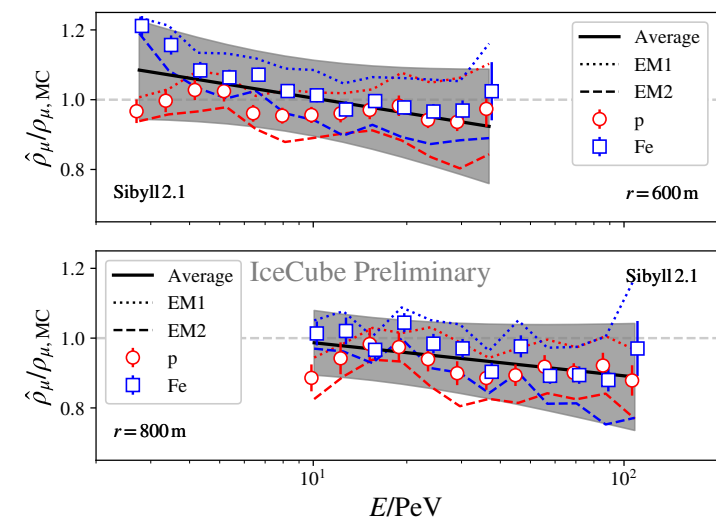

(a)
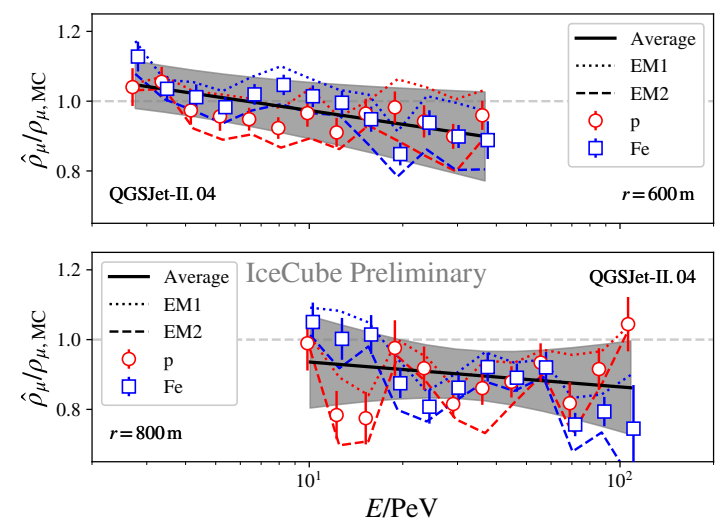

(c)
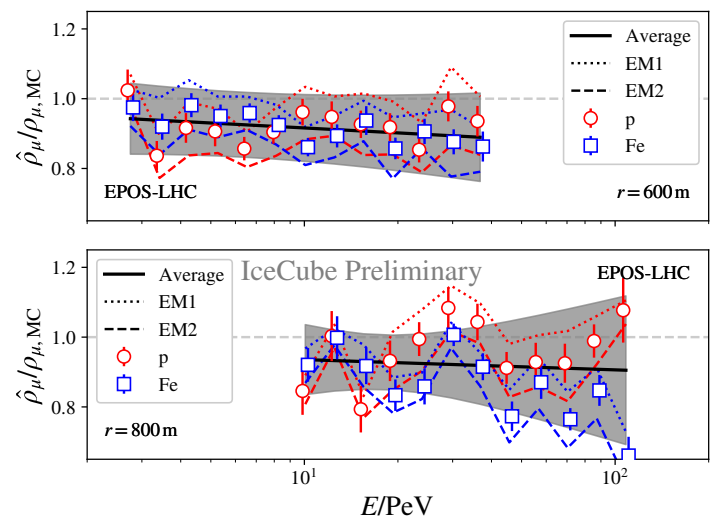

(b)
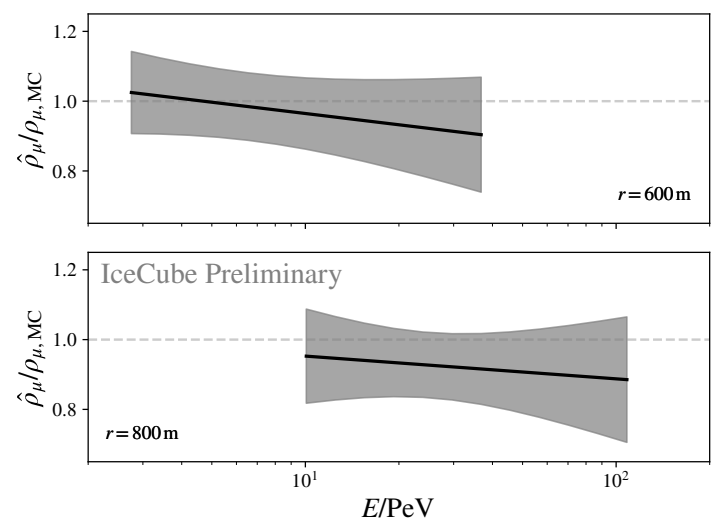

(d)

Figure 4: Ratio of raw reconstructed over true muon density at $600 \mathrm{~m}$ and $800 \mathrm{~m}$ lateral distance, as a function of reconstructed energy, for simulated air showers using the hadronic interaction models Sibyll 2.1 (a), EPOS-LHC (b), and QGSJet-II.04 (c). Squares and circles correspond to iron and proton simulated showers, dotted and dashed lines indicate the ratios obtained using two different EM models. Corresponding fits are shown as solid lines where the grey band represents the corresponding uncertainties. Figure (d) shows the corresponding average ratios for these three hadronic models.

The inverse of this ratio is used as an $M C$ correction factor to adjust the result in reconstructed data. However, as shown in Fig. 4, the correction factor depends on the mass composition of the sample, since iron primaries require a larger correction. The actual composition is unknown, so the correction factor applied to the data is the average of the proton and iron factors, with a systematic uncertainty of half of the difference. A linear fit to this average yields the correction factors for each hadronic model, depicted as a black lines in Fig. 4. There are three contributions to the uncertainty in the correction factor, depicted as a grey band: the electromagnetic signal model used (EM1/EM2), the assumed mass composition, and the statistical uncertainty in the fit. All these uncertainties appear as systematic uncertainties in the resulting muon density and they are further discussed in the following.

The systematic uncertainty in this analysis arises from four main sources which are described in detail in Ref. [8]. The uncertainty in the energy determination causes a systematic uncertainty of about $7 \%$ in the muon density because of the correlation between energy and muon number. In addition, the effect of the EM model assumption in the likelihood fit of the signal model introduces 
an uncertainty of up to about $10 \%$ which is estimated from the differences obtained using the two electromagnetic models EM1 and EM2, as shown in Fig. 4. As also shown in Fig. 4, the correction depends on the composition assumption in MC and the corresponding uncertainty is estimated to be half the difference of the correction for proton and iron. The limited statistics of the simulations also introduces an uncertainty which is included in the uncertainty when applying the MC correction. For the results derived using the average correction in Fig. 4d, the model differences are also accounted for in the uncertainties.

\section{Results}

The resulting muon densities at lateral distances of $600 \mathrm{~m}$ and $800 \mathrm{~m}$ for EAS energies from $2.5 \mathrm{PeV}$ to $40 \mathrm{PeV}$ and $9 \mathrm{PeV}$ to $120 \mathrm{PeV}$, respectively, after applying the average correction factors from Fig. 4d, are shown in Fig. 5. The predictions from simulations based on the hadronic interaction models Sibyll 2.1, EPOS-LHC, and QGSJet-II.04 for proton and iron shower are shown as red and blue lines. The corresponding muon densities after applying the individual correction factors from Figs. $4 \mathrm{a}$ to $4 \mathrm{c}$ for each hadronic interaction model separately are shown in Fig. 6 (left). Within their uncertainties, the measured muon densities are between the model predictions assuming proton and iron primaries. However, for the post-LHC mod-

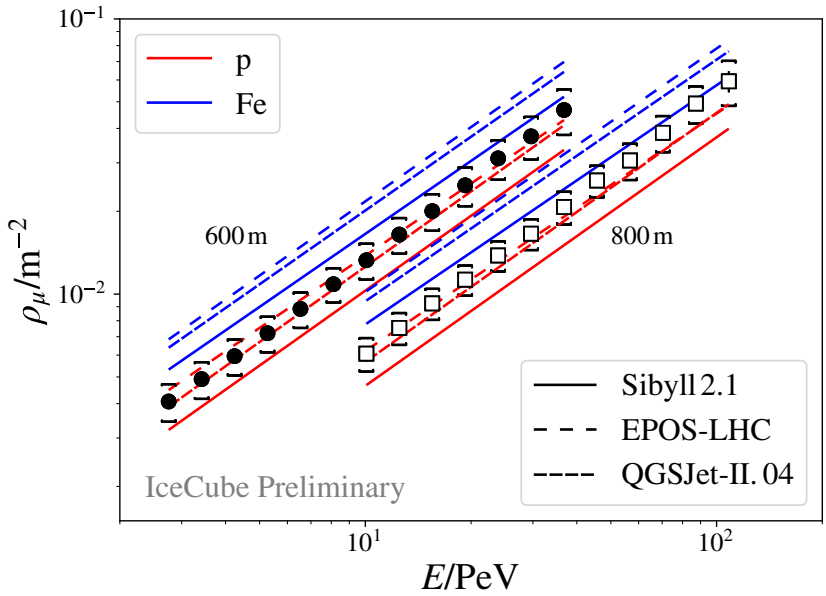

Figure 5: Measured muon density at $600 \mathrm{~m}$ (solid circles) and $600 \mathrm{~m}$ (white squares) lateral distance after applying the average correction from Fig. 4d. Error bars indicate the statistical, brackets the systematic uncertainty. Shown for comparison are the corresponding simulated densities for proton and iron (red and blue lines).

els the predictions suggest a very light composition at the lowest energies below $10 \mathrm{PeV}$, in particular using EPOS-LHC, which appears to be in tension with other experimental results $[1,12,13]$.

In order to compare the measured muon densities to predictions from different hadronic interaction models with certain cosmic ray flux assumptions in more detail, we define the quantity

$$
z=\frac{\log \left(\rho_{\mu}\right)-\log \left(\rho_{\mu, \mathrm{p}}\right)}{\log \left(\rho_{\mu, \mathrm{Fe}}\right)-\log \left(\rho_{\mu, \mathrm{p}}\right)},
$$

where $\rho_{\mu}$ is the measured muon density while $\rho_{\mu, \mathrm{p}}$ and $\rho_{\mu, \mathrm{Fe}}$ are the muon densities obtained from simulated proton and iron showers, respectively. The resulting distributions are shown in Fig. 6 (right), compared to predictions assuming the GSF [9], GST [14], and H3a [15] cosmic ray flux models. While Sibyll 2.1 describes the expected behaviour fairly well, at least up to energies of about $50 \mathrm{PeV}$, the post-LHC models predict more muons, yielding a very light composition. This is in tension with the model predictions which are based on and are within their uncertainties in agreement with experimental observations of the cosmic ray mass composition. 

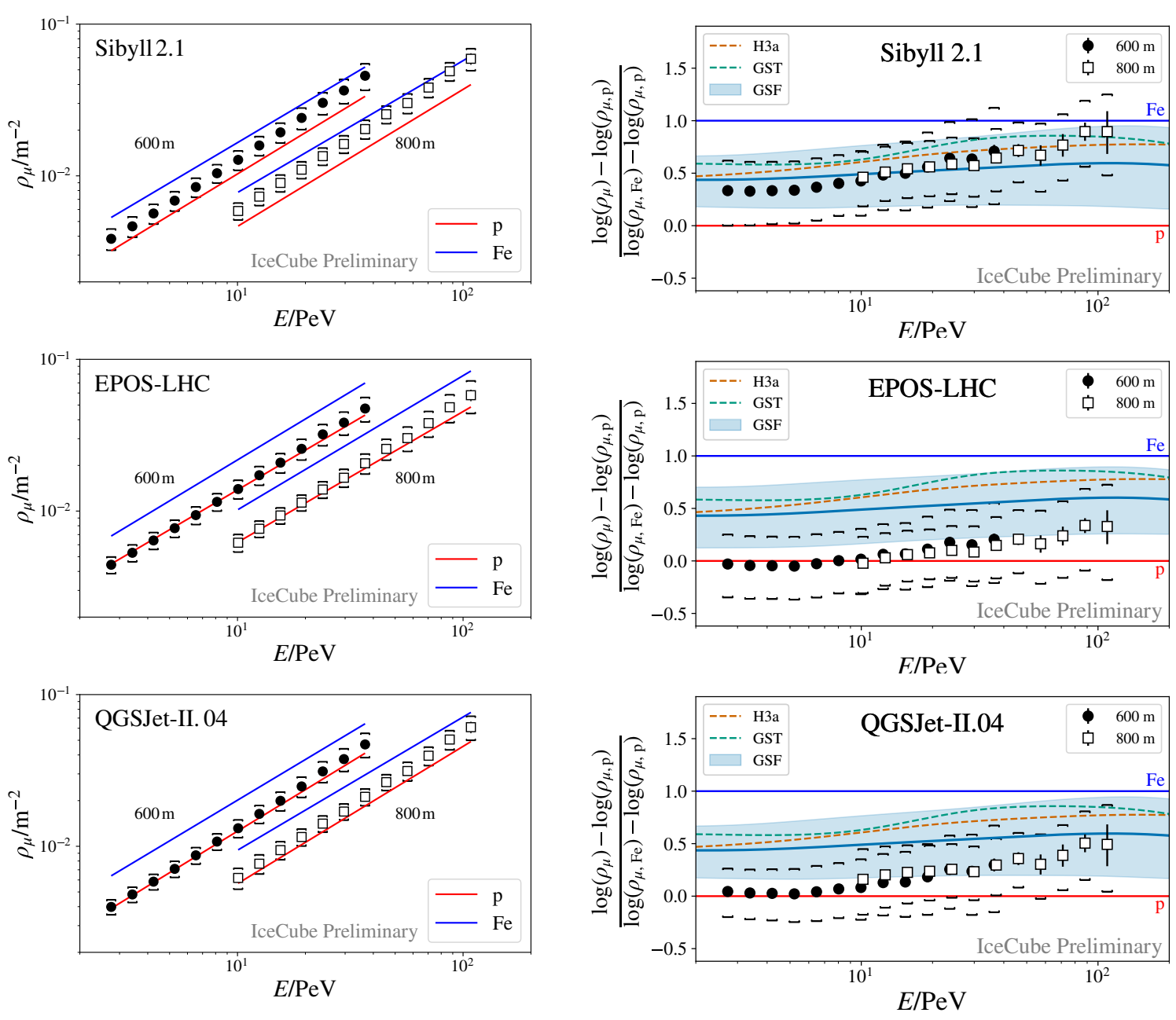

Figure 6: The final muon density measured in IceTop compared to predictions from the hadronic interaction models Sibyll 2.1 (top), EPOS-LHC (center), and QGSJet-II.04 (bottom). The figures on the right show the corresponding z-values, as defined in Eq. (1), as well as expectations from the cosmic ray flux models GSF (with error band), GST, and H3a. The error bars represent the statistical, brackets systematic uncertainties.

\section{Conclusions}

We have presented a measurement of the density of $\mathrm{GeV}$ muons at lateral distances of $600 \mathrm{~m}$ and $800 \mathrm{~m}$ for EAS energies from $2.5 \mathrm{PeV}$ to $40 \mathrm{PeV}$ and $9 \mathrm{PeV}$ to $120 \mathrm{PeV}$ in IceTop at an atmospheric depth of about $690 \mathrm{~g} / \mathrm{cm}^{2}$. While the measured muon densities agree within their uncertainties with predictions based on Sibyll 2.1, the post-LHC models predict too large average muon densities assuming realistic cosmic ray flux models consistent with experimental data. While the modeltuning to LHC data improves the agreement with measurements at the highest EAS energies our results suggest that the muon densities in post-LHC models are not correct for primary energies below approximately $100 \mathrm{PeV}$. However, a systematic shift in the reconstructed primary energy can also cause an apparent discrepancy in muon density. For this reason, a detailed comparison of results from multiple observatories across all energies is needed to understand the production of $\mathrm{GeV}$ muons in air showers, as shown in Refs. [16, 17]. 
Future coincident measurements with the in-ice detector of IceCube and IceTop enable the simultaneous detection of $\mathrm{GeV}$ and $\mathrm{TeV}$ muons in EAS which provides spectral information and will help to further test hadronic interaction models $[18,19]$. In addition, new surface extensions of the IceCube Neutrino Observatory [20] in the context of IceCube-Gen2 [21] will significantly increase the phase space of muon measurements towards larger zenith angles and higher EAS energies. This will allow measurements of the angular distribution of the atmospheric muon flux, up to EeV air shower energies, closing the gap towards EAS experiments at the highest energies.

\section{References}

[1] K.-H. Kampert and M. Unger Astropart. Phys. 35 (2012) 660-678.

[2] T. Pierog et al. Phys. Rev. C 92 no. 3, (2015) 034906.

[3] S. Ostapchenko Phys. Rev. D 83 (2011) 014018.

[4] E.-J. Ahn et al. Phys. Rev. D 80 (2009) 094003.

[5] IceCube Collaboration, R. Abbasi et al. Nucl. Instrum. Meth. A 700 (2013) 188-220.

[6] IceCube Collaboration, M. G. Aartsen et al. JINST 12 no. 03, (2017) P03012.

[7] IceCube Collaboration, M. G. Aartsen et al. Phys. Rev. D 88 no. 4, (2013) 042004.

[8] IceCube Collaboration, M. G. Aartsen et al. submitted to Phys. Rev. D (2021) .

[9] H. P. Dembinski et al. PoS ICRC2017 (2018) 533.

[10] IceCube Collaboration, J. G. Gonzalez EPJ Web Conf. 208 (2019) 03003.

[11] D. Heck et al., Report FZKA 6019, Forschungszentrum Karlsruhe, 1998.

[12] IceCube Collaboration, M. G. Aartsen et al. Phys. Rev. D 100 no. 8, (2019) 082002.

[13] KASCADE-Grande Collaboration, W. D. Apel et al. Astropart. Phys. 47 (2013) 54-66.

[14] T. K. Gaisser, T. Stanev, and S. Tilav Front. Phys.(Beijing) 8 (2013) 748-758.

[15] T. K. Gaisser Astropart. Phys. 35 (2012) 801-806.

[16] H. P. Dembinski et al. EPJ Web Conf. 210 (2019) 02004.

[17] D. Soldin et al. PoS ICRC2021 (2021) 349.

[18] IceCube Collaboration, S. De Ridder, E. Dvorak, and T. Gaisser PoS ICRC2017 (2017) 319.

[19] IceCube Collaboration, S. Verpoest PoS ICRC2021 (2021) 357.

[20] IceCube Collaboration, A. Haungs EPJ Web Conf. 210 (2019) 06009.

[21] IceCube-Gen2 Collaboration, M. G. Aartsen et al. J. Phys. G 48 no. 6, (2021) 060501. 


\section{Full Author List: IceCube Collaboration}

R. Abbasi ${ }^{17}$, M. Ackermann ${ }^{59}$, J. Adams ${ }^{18}$, J. A. Aguilar ${ }^{12}$, M. Ahlers ${ }^{22}$, M. Ahrens ${ }^{50}$, C. Alispach ${ }^{28}$, A. A. Alves Jr. ${ }^{31}$, N. M. $\mathrm{Amin}^{42}$, R. An ${ }^{14}$, K. Andeen ${ }^{40}$, T. Anderson ${ }^{56}$, G. Anton ${ }^{26}$, C. Argüelles ${ }^{14}$, Y. Ashida ${ }^{38}$, S. Axani ${ }^{15}$, X. Bai ${ }^{46}$, A. Balagopal V. ${ }^{38}$, A. Barbano ${ }^{28}$, S. W. Barwick ${ }^{30}$, B. Bastian ${ }^{59}$, V. Basu ${ }^{38}$, S. Baur ${ }^{12}$, R. Bay ${ }^{8}$, J. J. Beatty ${ }^{20,21}$, K.-H. Becker ${ }^{58}$, J. Becker Tjus ${ }^{11}$, C. Bellenghi $^{27}$, S. BenZvi ${ }^{48}$, D. Berley ${ }^{19}$, E. Bernardini ${ }^{59,60}$, D. Z. Besson ${ }^{34,61}$, G. Binder ${ }^{8,9}$, D. Bindig ${ }^{58}$, E. Blaufuss ${ }^{19}$, S. Blot ${ }^{59}$, M. Boddenberg ${ }^{1}$, F. Bontempo ${ }^{31}$, J. Borowka ${ }^{1}$, S. Böser ${ }^{39}$, O. Botner ${ }^{57}$, J. Böttcher ${ }^{1}$, E. Bourbeau ${ }^{22}$, F. Bradascio ${ }^{59}$, J. Braun ${ }^{38}$, S. Bron $^{28}$, J. Brostean-Kaiser ${ }^{59}$, S. Browne ${ }^{32}$, A. Burgman ${ }^{57}$, R. T. Burley ${ }^{2}$, R. S. Busse ${ }^{41}$, M. A. Campana ${ }^{45}$, E. G. Carnie-Bronca ${ }^{2}$, C. Chen ${ }^{6}$, D. Chirkin ${ }^{38}$, K. Choi ${ }^{52}$, B. A. Clark ${ }^{24}$, K. Clark ${ }^{33}$, L. Classen ${ }^{41}$, A. Colemann ${ }^{42}$, G. H. Collin ${ }^{15}$, J. M. Conrad ${ }^{15}$, P. Coppin $^{13}$, P. Correa ${ }^{13}$, D. F. Cowen ${ }^{55,56}$, R. Cross $^{48}$, C. Dappen ${ }^{1}$, P. Dave ${ }^{6}$, C. De Clercq ${ }^{13}$, J. J. DeLaunay ${ }^{56}$, H. Dembinski ${ }^{42}$, K. Deoskar $^{50}$, S. De Ridder ${ }^{29}$, A. Desai ${ }^{38}$, P. Desiati ${ }^{38}$, K. D. de Vries ${ }^{13}$, G. de Wasseige ${ }^{13}$, M. de With ${ }^{10}$, T. DeYoung ${ }^{24}$, S. Dharani ${ }^{1}$, A. Diaz ${ }^{15}$, J. C. Díaz-Vélez ${ }^{38}$, M. Dittmer ${ }^{41}$, H. Dujmovic ${ }^{31}$, M. Dunkman ${ }^{56}$, M. A. DuVernois ${ }^{38}$, E. Dvorak ${ }^{46}$, T. Ehrhardt ${ }^{39}$, P. Eller $^{27}$, R. Engel ${ }^{31,32}$, H. Erpenbeck ${ }^{1}$, J. Evans ${ }^{19}$, P. A. Evenson ${ }^{42}$, K. L. Fan ${ }^{19}$, A. R. Fazely ${ }^{7}$, S. Fiedlschuster ${ }^{26}$, A. T. Fienberg ${ }^{56}$, K. Filimonov ${ }^{8}$, C. Finley ${ }^{50}$, L. Fischer ${ }^{59}$, D. Fox ${ }^{55}$, A. Franckowiak ${ }^{11,59}$, E. Friedman ${ }^{19}$, A. Fritz ${ }^{39}$, P. Fürst ${ }^{1}$, T. K. Gaisser ${ }^{42}$, J. Gallagher $^{37}$, E. Ganster ${ }^{1}$, A. Garcia ${ }^{14}$, S. Garrappa ${ }^{59}$, L. Gerhardt ${ }^{9}$, A. Ghadimi ${ }^{54}$, C. Glaser ${ }^{57}$, T. Glauch ${ }^{27}$, T. Glüsenkamp ${ }^{26}$, A. Goldschmidt $^{9}$, J. G. Gonzalez ${ }^{42}$, S. Goswami ${ }^{54}$, D. Grant ${ }^{24}$, T. Grégoire ${ }^{56}$, S. Griswold ${ }^{48}$, M. Gündüz ${ }^{11}$, C. Günther ${ }^{1}$, C. Haack ${ }^{27}$,

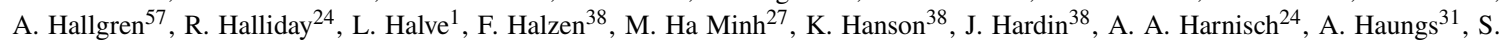
Hauser $^{1}$, D. Hebecker ${ }^{10}$, K. Helbing ${ }^{58}$, F. Henningsen ${ }^{27}$, E. C. Hettinger ${ }^{24}$, S. Hickford ${ }^{58}$, J. Hignight ${ }^{25}$, C. Hill ${ }^{16}$, G. C. Hill ${ }^{2}$, K. D.

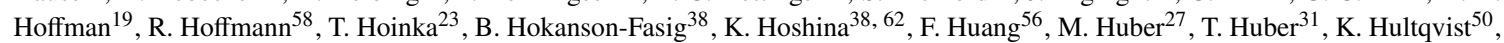
M. Hünnefeld ${ }^{23}$, R. Hussain ${ }^{38}$, S. In ${ }^{52}$, N. Iovine ${ }^{12}$, A. Ishihara ${ }^{16}$, M. Jansson ${ }^{50}$, G. S. Japaridze ${ }^{5}$, M. Jeong ${ }^{52}$, B. J. P. Jones ${ }^{4}$, D. Kang ${ }^{31}$, W. Kang ${ }^{52}$, X. Kang ${ }^{45}$, A. Kappes ${ }^{41}$, D. Kappesser ${ }^{39}$, T. Karg ${ }^{59}$, M. Karl ${ }^{27}$, A. Karle ${ }^{38}$, U. Katz ${ }^{26}$, M. Kauer ${ }^{38}$, M. Kellermann ${ }^{1}$, J. L. Kelley $^{38}$, A. Kheirandish ${ }^{56}$, K. Kin ${ }^{16}$, T. Kintscher ${ }^{59}$, J. Kiryluk ${ }^{51}$, S. R. Klein ${ }^{8,9}$, R. Koirala ${ }^{42}$, H. Kolanoski ${ }^{10}$, T. Kontrimas ${ }^{27}$, L. Köpke $^{39}$, C. Kopper ${ }^{24}$, S. Kopper ${ }^{54}$, D. J. Koskinen ${ }^{22}$, P. Koundal ${ }^{31}$, M. Kovacevich ${ }^{45}$, M. Kowalski ${ }^{10,59}$, T. Kozynets ${ }^{22}$, E. Kun ${ }^{11}$, N. Kurahashi ${ }^{45}$, N. Lad ${ }^{59}$, C. Lagunas Gualda ${ }^{59}$, J. L. Lanfranchi ${ }^{56}$, M. J. Larson ${ }^{19}$, F. Lauber ${ }^{58}$, J. P. Lazar ${ }^{14,38}$, J. W. Lee ${ }^{52}$, K. Leonard $^{38}$, A. Leszczyńska ${ }^{32}$, Y. Li ${ }^{56}$, M. Lincetto ${ }^{11}$, Q. R. Liu ${ }^{38}$, M. Liubarska ${ }^{25}$, E. Lohfink ${ }^{39}$, C. J. Lozano Mariscal ${ }^{41}$, L. Lu ${ }^{38}$,

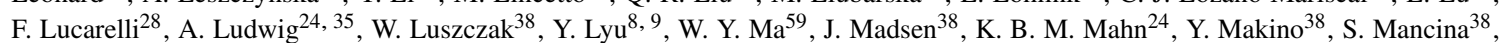

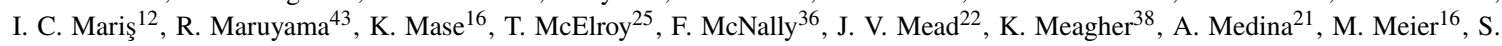
Meighen-Berger $^{27}$, J. Micallef ${ }^{24}$, D. Mockler ${ }^{12}$, T. Montaruli ${ }^{28}$, R. W. Moore ${ }^{25}$, R. Morse ${ }^{38}$, M. Moulai ${ }^{15}$, R. Naab ${ }^{59}$, R. Nagai ${ }^{16}$, U. Naumann $^{58}$, J. Necker ${ }^{59}$, L. V. Nguyễn ${ }^{24}$, H. Niederhausen ${ }^{27}$, M. U. Nisa ${ }^{24}$, S. C. Nowicki ${ }^{24}$, D. R. Nygren ${ }^{9}$, A. Obertacke Pollmann ${ }^{58}$, M. Oehler ${ }^{31}$, A. Olivas ${ }^{19}$, E. O'Sullivan ${ }^{57}$, H. Pandya ${ }^{42}$, D. V. Pankova ${ }^{56}$, N. Park ${ }^{33}$, G. K. Parker ${ }^{4}$, E. N. Paudel ${ }^{42}$, L. Paul ${ }^{40}$, C. Pérez de los $\operatorname{Heros}^{57}$, L. Peters ${ }^{1}$, J. Peterson ${ }^{38}$, S. Philippen ${ }^{1}$, D. Pieloth ${ }^{23}$, S. Pieper ${ }^{58}$, M. Pittermann ${ }^{32}$, A. Pizzuto ${ }^{38}$, M. Plum ${ }^{40}$, Y. Popovych $^{39}$, A. Porcelli ${ }^{29}$, M. Prado Rodriguez ${ }^{38}$, P. B. Price ${ }^{8}$, B. Pries ${ }^{24}$, G. T. Przybylski ${ }^{9}$, C. Raab ${ }^{12}$, A. Raissi ${ }^{18}$, M. Rameez $^{22}$, K. Rawlins $^{3}$, I. C. Rea ${ }^{27}$, A. Rehman ${ }^{42}$, P. Reichherzer ${ }^{11}$, R. Reimann ${ }^{1}$, G. Renzi ${ }^{12}$, E. Resconi ${ }^{27}$, S. Reusch ${ }^{59}$, W. Rhode ${ }^{23}$, M. Richman $^{45}$, B. Riedel ${ }^{38}$, E. J. Roberts ${ }^{2}$, S. Robertson ${ }^{8,9}$, G. Roellinghoff ${ }^{52}$, M. Rongen ${ }^{39}$, C. Rott ${ }^{49}, 52$, T. Ruhe ${ }^{23}$, D. Ryckbosch ${ }^{29}$, D. Rysewyk $\mathrm{Cantu}^{24}$, I. Safa ${ }^{14,38}$, J. Saffer ${ }^{32}$, S. E. Sanchez Herrera ${ }^{24}$, A. Sandrock ${ }^{23}$, J. Sandroos ${ }^{39}$, M. Santander ${ }^{54}$, S. Sarkar ${ }^{44}$, S. Sarkar ${ }^{25}$, K. Satalecka $^{59}$, M. Scharf ${ }^{1}$, M. Schaufel ${ }^{1}$, H. Schieler ${ }^{31}$, S. Schindler ${ }^{26}$, P. Schlunder ${ }^{23}$, T. Schmidt ${ }^{19}$, A. Schneider ${ }^{38}$, J. Schneider ${ }^{26}$, F. G. Schröder ${ }^{31,42}$, L. Schumacher ${ }^{27}$, G. Schwefer ${ }^{1}$, S. Sclafani ${ }^{45}$, D. Seckel ${ }^{42}$, S. Seunarine ${ }^{47}$, A. Sharma ${ }^{57}$, S. Shefali ${ }^{32}$, M. Silva $^{38}$, B. Skrzypek $^{14}$, B. Smithers ${ }^{4}$, R. Snihur ${ }^{38}$, J. Soedingrekso ${ }^{23}$, D. Soldin ${ }^{42}$, C. Spannfellner ${ }^{27}$, G. M. Spiczak ${ }^{47}$, C. Spiering ${ }^{59,61}$, J. Stachurska $^{59}$, M. Stamatikos ${ }^{21}$, T. Stanev ${ }^{42}$, R. Stein ${ }^{59}$, J. Stettner ${ }^{1}$, A. Steuer ${ }^{39}$, T. Stezelberger ${ }^{9}$, T. Stürwald ${ }^{58}$, T. Stuttard ${ }^{22}$, G. W. Sullivan $^{19}$, I. Taboada ${ }^{6}$, F. Tenholt ${ }^{11}$, S. Ter-Antonyan ${ }^{7}$, S. Tilav ${ }^{42}$, F. Tischbein ${ }^{1}$, K. Tollefson $^{24}$, L. Tomankova ${ }^{11}$, C. Tönnis ${ }^{53}$, S.

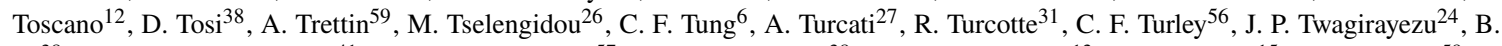
$\mathrm{Ty}^{38}$, M. A. Unland Elorrieta ${ }^{41}$, N. Valtonen-Mattila ${ }^{57}$, J. Vandenbroucke ${ }^{38}$, N. van Eijndhoven ${ }^{13}$, D. Vannerom ${ }^{15}$, J. van Santen ${ }^{59}, \mathrm{~S}$. Verpoest $^{29}$, M. Vraeghe ${ }^{29}$, C. Walck ${ }^{50}$, T. B. Watson ${ }^{4}$, C. Weaver ${ }^{24}$, P. Weigel ${ }^{15}$, A. Weindl ${ }^{31}$, M. J. Weiss ${ }^{56}$, J. Weldert ${ }^{39}$, C. Wendt $^{38}$, J. Werthebach ${ }^{23}$, M. Weyrauch ${ }^{32}$, N. Whitehorn ${ }^{24,35}$, C. H. Wiebusch ${ }^{1}$, D. R. Williams ${ }^{54}$, M. Wolf ${ }^{27}$, K. Woschnagg ${ }^{8}$, G. Wrede ${ }^{26}$, J. Wulff ${ }^{11}$, X. W. Xu $u^{7}$, Y. Xu ${ }^{51}$, J. P. Yanez ${ }^{25}$, S. Yoshida ${ }^{16}$, S. Yu ${ }^{24}$, T. Yuan ${ }^{38}$, Z. Zhang ${ }^{51}$

${ }^{1}$ III. Physikalisches Institut, RWTH Aachen University, D-52056 Aachen, Germany

2 Department of Physics, University of Adelaide, Adelaide, 5005, Australia

${ }^{3}$ Dept. of Physics and Astronomy, University of Alaska Anchorage, 3211 Providence Dr., Anchorage, AK 99508, USA

${ }^{4}$ Dept. of Physics, University of Texas at Arlington, 502 Yates St., Science Hall Rm 108, Box 19059, Arlington, TX 76019, USA

5 CTSPS, Clark-Atlanta University, Atlanta, GA 30314, USA

${ }^{6}$ School of Physics and Center for Relativistic Astrophysics, Georgia Institute of Technology, Atlanta, GA 30332, USA

${ }^{7}$ Dept. of Physics, Southern University, Baton Rouge, LA 70813, USA

${ }^{8}$ Dept. of Physics, University of California, Berkeley, CA 94720, USA

${ }^{9}$ Lawrence Berkeley National Laboratory, Berkeley, CA 94720, USA

${ }^{10}$ Institut für Physik, Humboldt-Universität zu Berlin, D-12489 Berlin, Germany

${ }^{11}$ Fakultät für Physik \& Astronomie, Ruhr-Universität Bochum, D-44780 Bochum, Germany

12 Université Libre de Bruxelles, Science Faculty CP230, B-1050 Brussels, Belgium

13 Vrije Universiteit Brussel (VUB), Dienst ELEM, B-1050 Brussels, Belgium

14 Department of Physics and Laboratory for Particle Physics and Cosmology, Harvard University, Cambridge, MA 02138, USA

15 Dept. of Physics, Massachusetts Institute of Technology, Cambridge, MA 02139, USA 
${ }^{16}$ Dept. of Physics and Institute for Global Prominent Research, Chiba University, Chiba 263-8522, Japan

${ }^{17}$ Department of Physics, Loyola University Chicago, Chicago, IL 60660, USA

${ }^{18}$ Dept. of Physics and Astronomy, University of Canterbury, Private Bag 4800, Christchurch, New Zealand

${ }^{19}$ Dept. of Physics, University of Maryland, College Park, MD 20742, USA

${ }^{20}$ Dept. of Astronomy, Ohio State University, Columbus, OH 43210, USA

${ }^{21}$ Dept. of Physics and Center for Cosmology and Astro-Particle Physics, Ohio State University, Columbus, OH 43210, USA

${ }^{22}$ Niels Bohr Institute, University of Copenhagen, DK-2100 Copenhagen, Denmark

${ }^{23}$ Dept. of Physics, TU Dortmund University, D-44221 Dortmund, Germany

${ }^{24}$ Dept. of Physics and Astronomy, Michigan State University, East Lansing, MI 48824, USA

${ }^{25}$ Dept. of Physics, University of Alberta, Edmonton, Alberta, Canada T6G 2E1

${ }^{26}$ Erlangen Centre for Astroparticle Physics, Friedrich-Alexander-Universität Erlangen-Nürnberg, D-91058 Erlangen, Germany

${ }^{27}$ Physik-department, Technische Universität München, D-85748 Garching, Germany

${ }^{28}$ Département de physique nucléaire et corpusculaire, Université de Genève, CH-1211 Genève, Switzerland

${ }^{29}$ Dept. of Physics and Astronomy, University of Gent, B-9000 Gent, Belgium

${ }^{30}$ Dept. of Physics and Astronomy, University of California, Irvine, CA 92697, USA

${ }^{31}$ Karlsruhe Institute of Technology, Institute for Astroparticle Physics, D-76021 Karlsruhe, Germany

${ }^{32}$ Karlsruhe Institute of Technology, Institute of Experimental Particle Physics, D-76021 Karlsruhe, Germany

${ }^{33}$ Dept. of Physics, Engineering Physics, and Astronomy, Queen's University, Kingston, ON K7L 3N6, Canada

${ }^{34}$ Dept. of Physics and Astronomy, University of Kansas, Lawrence, KS 66045, USA

${ }^{35}$ Department of Physics and Astronomy, UCLA, Los Angeles, CA 90095, USA

${ }^{36}$ Department of Physics, Mercer University, Macon, GA 31207-0001, USA

${ }^{37}$ Dept. of Astronomy, University of Wisconsin-Madison, Madison, WI 53706, USA

${ }^{38}$ Dept. of Physics and Wisconsin IceCube Particle Astrophysics Center, University of Wisconsin-Madison, Madison, WI 53706, USA

${ }^{39}$ Institute of Physics, University of Mainz, Staudinger Weg 7, D-55099 Mainz, Germany

${ }^{40}$ Department of Physics, Marquette University, Milwaukee, WI, 53201, USA

${ }^{41}$ Institut für Kernphysik, Westfälische Wilhelms-Universität Münster, D-48149 Münster, Germany

42 Bartol Research Institute and Dept. of Physics and Astronomy, University of Delaware, Newark, DE 19716, USA

${ }^{43}$ Dept. of Physics, Yale University, New Haven, CT 06520, USA

${ }^{44}$ Dept. of Physics, University of Oxford, Parks Road, Oxford OX1 3PU, UK

${ }^{45}$ Dept. of Physics, Drexel University, 3141 Chestnut Street, Philadelphia, PA 19104, USA

${ }^{46}$ Physics Department, South Dakota School of Mines and Technology, Rapid City, SD 57701, USA

${ }^{47}$ Dept. of Physics, University of Wisconsin, River Falls, WI 54022, USA

${ }^{48}$ Dept. of Physics and Astronomy, University of Rochester, Rochester, NY 14627, USA

${ }^{49}$ Department of Physics and Astronomy, University of Utah, Salt Lake City, UT 84112, USA

50 Oskar Klein Centre and Dept. of Physics, Stockholm University, SE-10691 Stockholm, Sweden

${ }^{51}$ Dept. of Physics and Astronomy, Stony Brook University, Stony Brook, NY 11794-3800, USA

52 Dept. of Physics, Sungkyunkwan University, Suwon 16419, Korea

${ }^{53}$ Institute of Basic Science, Sungkyunkwan University, Suwon 16419, Korea

${ }^{54}$ Dept. of Physics and Astronomy, University of Alabama, Tuscaloosa, AL 35487, USA

${ }^{55}$ Dept. of Astronomy and Astrophysics, Pennsylvania State University, University Park, PA 16802, USA

56 Dept. of Physics, Pennsylvania State University, University Park, PA 16802, USA

${ }^{57}$ Dept. of Physics and Astronomy, Uppsala University, Box 516, S-75120 Uppsala, Sweden

${ }^{58}$ Dept. of Physics, University of Wuppertal, D-42119 Wuppertal, Germany

${ }^{59}$ DESY, D-15738 Zeuthen, Germany

${ }^{60}$ Università di Padova, I-35131 Padova, Italy

${ }^{61}$ National Research Nuclear University, Moscow Engineering Physics Institute (MEPhI), Moscow 115409, Russia

${ }^{62}$ Earthquake Research Institute, University of Tokyo, Bunkyo, Tokyo 113-0032, Japan

\section{Acknowledgements}

USA - U.S. National Science Foundation-Office of Polar Programs, U.S. National Science Foundation-Physics Division, U.S. National Science Foundation-EPSCoR, Wisconsin Alumni Research Foundation, Center for High Throughput Computing (CHTC) at the University of Wisconsin-Madison, Open Science Grid (OSG), Extreme Science and Engineering Discovery Environment (XSEDE), Frontera computing project at the Texas Advanced Computing Center, U.S. Department of Energy-National Energy Research Scientific Computing Center, Particle astrophysics research computing center at the University of Maryland, Institute for Cyber-Enabled Research at Michigan State University, and Astroparticle physics computational facility at Marquette University; Belgium - Funds for Scientific Research (FRS-FNRS and FWO), FWO Odysseus and Big Science programmes, and Belgian Federal Science Policy Office (Belspo); Germany - Bundesministerium für Bildung und Forschung (BMBF), Deutsche Forschungsgemeinschaft (DFG), Helmholtz Alliance for Astroparticle Physics (HAP), Initiative and Networking Fund of the Helmholtz Association, Deutsches Elektronen Synchrotron (DESY), and High Performance Computing cluster of the RWTH Aachen; Sweden - Swedish Research Council, Swedish Polar Research Secretariat, Swedish National Infrastructure for Computing (SNIC), and Knut and Alice Wallenberg Foundation; Australia - Australian 
Research Council; Canada - Natural Sciences and Engineering Research Council of Canada, Calcul Québec, Compute Ontario, Canada Foundation for Innovation, WestGrid, and Compute Canada; Denmark - Villum Fonden and Carlsberg Foundation; New Zealand Marsden Fund; Japan - Japan Society for Promotion of Science (JSPS) and Institute for Global Prominent Research (IGPR) of Chiba University; Korea - National Research Foundation of Korea (NRF); Switzerland - Swiss National Science Foundation (SNSF); United Kingdom - Department of Physics, University of Oxford. 